\title{
Many-electron, many-photon theory of nonlinear polarizabilities
}

\author{
C. A. Nicolaides, Th. Mercouris, and G. Aspromallis \\ Theoretical and Physical Chemistry Institute, National Hellenic Research Foundation, 48 Vas. Constantinou \\ Avenue, Athens 116 35, Greece
}

Received September 15, 1989; accepted December 13, 1989

\begin{abstract}
We present a many-electron, many-photon (MEMP) nonperturbative theory of nonlinear static and dynamic polarizabilities of polyelectronic atoms and molecules. The time-dependent, dynamic polarization is treated as a frequency-dependent problem with the Floquet assumption that the computed quantities are averaged over a cycle. The MEMP theory defines a non-Hermitian problem whose efficient solution yields a complex eigenvalue to all orders in the interelectronic and the electron ac or dc field interactions. When the real part is subtracted from the unperturbed energy of the free state, we are left with the frequency-dependent total energy shift $\Delta(\omega)$. This is then fitted to a polynomial expansion whose coefficients are the required polarizabilities representing the induced polarization averaged over a cycle. Results are given for the $\alpha, \alpha(\omega)$, and $\gamma, \gamma(\omega)$ of $\mathrm{H}$ and of $\mathrm{Li}^{-}$. For $\mathrm{Li}^{-}$, only $\alpha$ had been computed earlier by many-electron approaches. The dynamic $\alpha(\omega)$ was computed before in a quasi-classical approximation with a simple wave function. Comparison shows a qualitative agreement but not a quantitative one.
\end{abstract}

\section{INTRODUCTION}

When an atomic or a molecular electronic state is placed in a dc or an ac field, it suffers an energy shift $(\Delta E)$ and has an energy width $(\Gamma)$ that is caused by its ionization either through tunneling or through direct single-photon or multiphoton ionization.

The physics of these phenomena is usually described in terms of notions of perturbation theory. This leads to a series expansion of $\Delta E$ or $\Gamma$ as a function of field strength. When such an expansion is made, the implicit expectation is that the series can be computed term by term and that it converges or that it can somehow be summed. (This is more easily said than done, especially for polyelectronic systems.) In practice, convergence of the series is not guaranteed, and advanced methods have been devised for the study of such expansions to high orders. Results of high numerical accuracy of related computations for the one-electron systems $\mathrm{H}$ and $\mathrm{H}_{2}{ }^{+}$can be found in Ref. 1 (for dc and ac field energy shifts) and in Ref. 2 for dc field widths.

For systems with more than one electron, calculation of the field-induced $\Delta E$ and $\Gamma$ to high order through perturbation-theory-based expansions has not been accomplished to our knowledge. The existing variation-perturbation methods for the computation of the series coefficients for $\Delta E$, called linear and nonlinear, static, or dynamic polarizabilities, ${ }^{3-11}$ or the so-called lowest-order perturbation theory for the computation of the multiphoton ionization rates (valid only for weak fields), ${ }^{12}$ have led to polyelectronic computations of only the first one or two terms and with varying degress of accuracy with respect to the effects of interelectronic interactions. A complete and reliable theory of dynamic linear and nonlinear atomic polarizabilities (i.e., of the frequency-dependent coefficients in the expansion of $\Delta E)$ must be based on a general, as well as implementable, formalism, which permits the treatment of the many-elec- tron, many-photon (MEMP) problem to all orders and without any restrictions as regards subtleties of atomic structure.

The MEMP theory of dynamic polarizabilities that is presented below and its application to the four-electron anion, $\mathrm{Li}^{-}$have been developed with the aforementioned desiderata in mind. In particular, this is the first time to the authors' knowledge that the nonlinear polarizability $\gamma(\omega)$ has been computed rigorously for an atomic system with more than two electrons.

\section{LINEAR $(\alpha)$ AND NONLINEAR $(\gamma)$ POLARIZABILITIES OF NEGATIVE IONS: THEORETICAL ASPECTS AND PREVIOUS APPROACHES AND RESULTS}

For negative ions, even if one forgets the questions of convergence of a perturbation expansion that arise much earlier than in the case of neutrals, the accurate calculation of properties such as dipole polarizabilities $(\alpha)$ and hyperpolarizabilities $(\gamma)$, whether static or dynamic, poses serious challenges to theory. This is because, apart from the general problem of treating the atomic and molecular structure correctly, there are distinct differences between negative ions and corresponding neutral or ionized species. From the point of view of charge distribution and induced polarization, the linear and especially the nonlinear polarizabilities are sensitive to small and diffuse components present in the wave functions of negative ions. This sensitivity is significantly reduced in the neutrals, for which the analogous components as well as the various polarizabilities are much smaller. The implication of this fact is that for closed-shell atoms and molecules calculations at the coupled HartreeFock (CHF) (or, equivalently, at the time-dependent Hartree-Fock or the random-phase) approximation, which do not include correlation corrections, yield reasonable results; for negative ions, however, such predictions are unreliable. 
For example, consider the situation with the $\alpha$ of $\mathrm{H}^{-}$and of He. ${ }^{9,13}$ For $\mathrm{H}^{-}, \alpha(\mathrm{CHF})=93$ a.u. and $\alpha$ (exact) $=206$ a.u. On the other hand, for $\mathrm{He}, \alpha(\mathrm{CHF})=1.32$ a.u. and $\alpha$ (exact) $=1.38$ a.u. ${ }^{14}$

Looking at the problem from a different angle, we see that the negative ions have all their oscillator strength in the continuous spectrum, and theory must account for this fact consistently. For example, now the decoupling from higherorder effects, implied in all the theories that compute the coefficients of the $\Delta E$ series [see Eq. (1) below] to the corresponding order of perturbation theory in the field, need not be complete. On the contrary, for many neutral or ionized atoms, such as the alkalis and the alkaline earths, most of the oscillator strength comes from the first two or three excited states, whose good wave-function representation, in addition to that of the ground state, secures reasonably accurate linear polarizability calculations. ${ }^{16}$

The theory that is presented and applied here to the computation of linear and nonlinear polarizabilities of $\mathrm{Li}^{-}$incorporates the important effects of electron-electron and electron-field interactions to all orders and yields both $\Delta E$ and $\Gamma$.

With respect to the width, the first results from advanced calculations on negative ions were presented elsewhere. ${ }^{17,18}$

What is the situation regarding the available theories and computations for atoms and especially negative ions?

Being two-electron systems, and therefore treatable with $r_{i j}$-dependent (Hylleraas) basis sets, $\mathrm{H}^{-}$and its isoelectronic closed shell He have been subjected to a plethora of computational investigations, which include electron correlation (see Tables 1 and 2). The reliability of such computations for the static $\alpha$ is high. ${ }^{9}$ However, for $\gamma$ only one value exists (see Ref. 5 and Table 2). Even though a 150-term Hylleraas wave function was employed in the variation-perturbation scheme of computing linear and nonlinear polarizabilities, the result was considered unreliable by the authors, a fact that indicates the sensitivity of calculations of nonlinear quantities in negative ions to function spaces.

For systems with more than two electrons, a theory must be developed along directions that can analyze and compute the general many-electron-field interaction problem.

Recently, ${ }^{21,24,30,38}$ results from extensive calculations on static $\alpha$ and $\gamma$ of $\mathrm{Li}^{-},{ }^{24} \mathrm{~F}^{-}, 21,30$ and $\mathrm{Cl}^{-}$(Ref. 38) were published. Accurate knowledge of these quantities is essential for the reliable construction of ion-atom interaction potentials $^{21,37}$ or of crystal properties. ${ }^{39}$ The calculations of Refs. $21,24,30$, and 38 have employed powerful methods of the type by which much of the computational quantum chemistry of the past two decades is being carried out. Such methods are the many-body perturbation theory to order $n$ $[\operatorname{MBPT}(n)]$, full configuration interaction, coupled cluster, and Green's-function-type expansions. The underlying characteristic of these approaches is the use of large orthonormal basis sets of Gaussian- or Slater-type orbitals, which are common to the zeroth-order function (often restricted by the computational algorithms to be a single determinant) and to the function space describing correlation- or fieldinduced effects. Aspects of the problem of reliably computing the electric properties of polyelectronic systems in the framework of the aforementioned theories can be found in Refs. 11, 22, 26, and 32 .

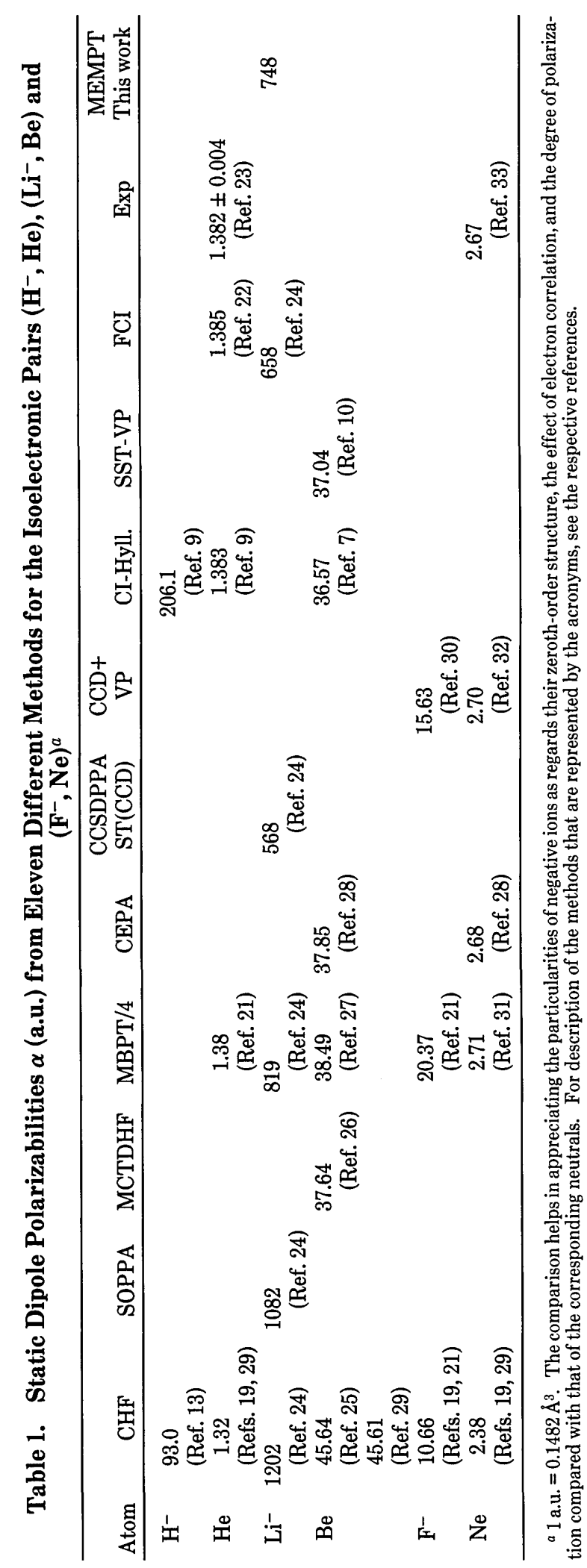


Table 2. Static Hyperpolarizabilities $\gamma(\text { a.u. })^{a}$

\begin{tabular}{|c|c|c|c|c|c|c|c|}
\hline Atom & $\mathrm{CHF}$ & MBPT(4) & $\mathrm{CCD}+\mathrm{ST}(\mathrm{CCD})$ & $\begin{array}{c}\text { CI-Hyll. } \\
\text { VP }\end{array}$ & Exper. & $\mathrm{FCI}$ & $\begin{array}{c}\text { MEMPT } \\
\text { (This Work) }\end{array}$ \\
\hline $\mathrm{H}^{-}$ & & & & $\begin{array}{l}1.74 \times 10^{7} \\
\text { (Ref. 5) }\end{array}$ & & & \\
\hline $\mathrm{He}$ & $\begin{array}{l}35.3 \\
\text { (Ref. 21) } \\
35.8 \\
\text { (Ref. 29) }\end{array}$ & $\begin{array}{c}42.05 \\
\text { (Ref. 21) }\end{array}$ & & $\begin{array}{l}42.8 \\
\quad \text { (Ref. 5) } \\
41.9 \\
\quad \text { (Ref. 34) } \\
43.1 \\
\quad \text { (Ref. 36) }\end{array}$ & $\begin{array}{l}51.6 \pm 8 \\
\text { (Ref. 35) }\end{array}$ & $\begin{array}{l}43.7 \\
\quad \text { (Ref. 22) }\end{array}$ & \\
\hline $\mathrm{Li}^{-}$ & $\begin{array}{l}41900 \\
\quad \text { (Ref. 20) }\end{array}$ & & & & & & $5.1 \times 10^{8}$ \\
\hline $\mathrm{Be}$ & $\begin{array}{l}39900 \\
\text { (Ref. 29) } \\
38760 \\
\text { (Ref. 32) }\end{array}$ & $\begin{array}{l}33260 \\
\quad \text { (Ref. 32) }\end{array}$ & $\begin{array}{l}31480 \\
\quad \text { (Ref. 32) }\end{array}$ & & & & \\
\hline $\mathrm{F}^{-}$ & $\begin{array}{l}11400 \\
\quad \text { (Ref. 21) }\end{array}$ & $\begin{array}{l}78000 \\
\quad \text { (Ref. 21) }\end{array}$ & & & & & \\
\hline $\mathrm{Ne}$ & $\begin{array}{l}70.0 \\
\text { (Refs. 32, 29) }\end{array}$ & $\begin{array}{l}104.6 \\
\text { (Ref. 31) }\end{array}$ & $\begin{array}{l}113.9+9.1 \\
\text { (Ref. 32) }\end{array}$ & & $\begin{array}{l}116 \pm 2 \\
\quad \text { (Ref. 37) }\end{array}$ & & \\
\hline
\end{tabular}

${ }^{a}$ Note that the research of Ref. 5 on $\mathrm{H}^{-}$and $\mathrm{He}$ used 150-term Hylleraas wave functions. However, the authors consider their $\gamma$ value for $\mathrm{H}^{-}$unreliable.

One interesting feature of the content of Refs. 11, 21, 24, 30 , and 34 and of the research to which they refer is the apparent expectation that MBPT(4), whenever it is applicable, is a convenient procedure for the reliable computation of static $\alpha$ and $\gamma$ (dynamic $\alpha$ and $\gamma$ are not reported for these negative ions). The extent to which this assertion is valid can be deduced from Tables 1 and 2, where we have collected results from various theories on $\mathrm{H}^{-}, \mathrm{Li}^{-}$, and $\mathrm{F}^{-}$as well as on isoelectronic $\mathrm{He}, \mathrm{Be}$, and $\mathrm{Ne}$. It can be seen that when zeroth-order correlation is strong or when it is applied to negative ions, MBPT(4) is not accurate. Within the conventional computational approaches, the theory must then go to higher orders in the hierarchy of algorithms (e.g., multireference coupled cluster or full configuration interaction). In fact, this is also the conclusion of Kucharski et al. ${ }^{30}$ However, going beyond MBPT(4) is rather cumbersome.

The brief considerations above may serve as a justification for the need to have a practical polyelectronic theory of polarizabilities that is nonperturbative and that pays attention to electronic structure characteristics and to suitably adjusted function spaces. The possibility of efficient calculations of polarizabilities is indicated by our variation-perturbation results on the $\alpha$ of $\mathrm{Be} .{ }^{10}$ This alternative approach emphasized the optimized representation of the important correlation effects in the spirit of the state-specific theory ${ }^{40}$ and resulted in a much smaller overall calculation while yielding a value that is in essential agreement with that of the accurate calculation of Sims and Rumble ${ }^{7}$ (Table 1). ${ }^{41}$

\section{THEORY}

The energy shift $\Delta$, which is induced in the atomic state by an external dc field of strength $F_{0}$ or a linearly polarized ac field, $F_{0} \cos \omega t$, can be expanded in a Taylor series as

$$
\Delta(\text { static })=-\frac{1}{2 !} \alpha F_{0}{ }^{2}-\frac{1}{4 !} \gamma F_{0}^{4}-\frac{1}{6 !} \delta F_{0}{ }^{6}-\ldots
$$

and

$$
\begin{aligned}
\Delta(\omega)= & -\frac{1}{2 !} \alpha(\omega) \overline{\left(F_{0} \cos \omega t\right)^{2}}-\frac{1}{4 !} \gamma(\omega) \overline{\left(F_{0} \cos \omega t\right)^{4}}-\ldots \\
= & -\frac{1}{2 !} \alpha(\omega)\left(\frac{1}{2}\right){F_{0}}^{2}-\frac{1}{4 !} \gamma(\omega)\left(\frac{3}{8}\right) F_{0}^{4} \\
& -\frac{1}{6 !} \delta(\omega)\left(\frac{5}{16}\right) F_{0}^{6}-\ldots
\end{aligned}
$$

Equation (1) is the well-known definition of the linear $(\alpha)$ and the nonlinear ( $\gamma$, etc.) static polarizabilities of atoms and of symmetric molecules. ${ }^{3}$

The dynamic energy shift, $\Delta(\omega)$, is expanded in an analogous form. As written, $\alpha(\omega)$ and $\gamma(\omega)$ represent the induced polarization averaged over an optical cycle. ${ }^{42}$

For perturbation-theory-dependent theories, the linear and nonlinear coefficients of the series of Eqs. (1)-(3) are obtained (in principle) individually from corresponding expressions. On the other hand, the present nonperturbative MEMP theory incorporates the interelectronic and fieldinduced effects to all orders, and the related calculation yields directly $\Delta$ and $\Delta(\omega)$. These quantities may then be computed for small field values and fitted to a polynomial of a high degree so as to produce stable results for $\alpha$ and $\gamma$.

Let us consider the (more difficult) ac field problem. We obtain $\Delta$ from the solution of the time-independent, complex-eigenvalued, non-Hermitian equation ${ }^{17,18,43}$

$$
\left(H-z_{0}\right) \Psi=0,
$$

where

$$
H=H_{\text {atom }}+\hbar \omega \alpha_{\omega}{ }^{+} \alpha_{\omega}-\frac{F_{0}}{2} \sum_{i}^{N \text { electron }} z_{i}\left(\alpha_{\omega}{ }^{+}+\alpha_{\omega}\right)
$$


for linear polarization along the $\mathrm{z}$ axis, where $\alpha^{+}$and $\alpha_{\omega}$ are photon creation and annihilation operators, respectively, or

$$
H=H_{\text {atom }}-F_{0} \sum_{i}^{N \text { electron }} z_{i},
$$

for a static field along the $z$ axis,

$$
z_{0}\left(F_{0}, \omega\right)=E_{0}+\Delta\left(F_{0}, \omega\right)-i / 2 \Gamma\left(F_{0}, \omega\right) .
$$

The eigenvalue $z_{0}\left(F_{0}, \omega\right)$ represents an average over a cycle, in line with the definition of Eq. (2). $\psi$ is the nonnormalizable resonance function, which emerges naturally as a result of the perturbation-induced mixing of a bare, $N$-electron discrete level $\left(\psi_{0}, E_{0}\right)$ with the continuum of the ionized states $U(E) .{ }^{44}$ It consists of localized as well as of asymptotic components, which are made square integrable by the use of complex coordinates, $\rho=r e^{i \theta} .43-48$

In analogy with the problem of autoionization, the solution of Eq. (4) can be effected, in principle, by the diagonalization of the rotated Hamiltonian in a complete Hilbert space of orthonormal basis. 45,46 However, the conceptual as well as computational limitations of the conventional complex coordinate rotation method, which are known from the field of multichannel resonances in $N$-electron systems, ${ }^{48,49}$ are also present-even more severely-in the study of atoms perturbed by external fields.

The present MEMP theory avoids the complex coordinate rotation bottleneck and unsuitability for computing $\Delta$ by isolating in the complex plane the important correlation effects that contribute to the initial bound states from the contribution of the scattering functions that interact with the bound states by means of the external field and cause the energy shift and width. Formally, having changed the asymptotic boundary condition of Eq. (4) through $\rho=r e^{i \theta}$, we express the square-integrable solution $\hat{\Psi}(\rho)$ which is connected adiabatically to the unperturbed atomic $\left|\Psi_{0}\right\rangle$ as

$$
\left|\hat{\Psi}_{0}(\rho)\right\rangle=\sum_{i, n} a_{i, n}(\theta)\left|\Psi_{i}(\rho) ; n\right\rangle+\sum_{j, n} b_{j, n}(\theta)\left|X_{j}(\rho) ; n\right\rangle,
$$

where $\Psi_{i}$ denotes bound states, $X_{j}$ denotes the $L^{2}$ scattering states, and $n$ denotes the photon states. When the basis set of Eq. (8) is used, the eigenvalue Eq. (4) takes the MEMP matrix form ${ }^{50}$

$$
\tilde{\mathbf{H}}_{s} \mathbf{X}=z_{0} \mathbf{X}
$$

where

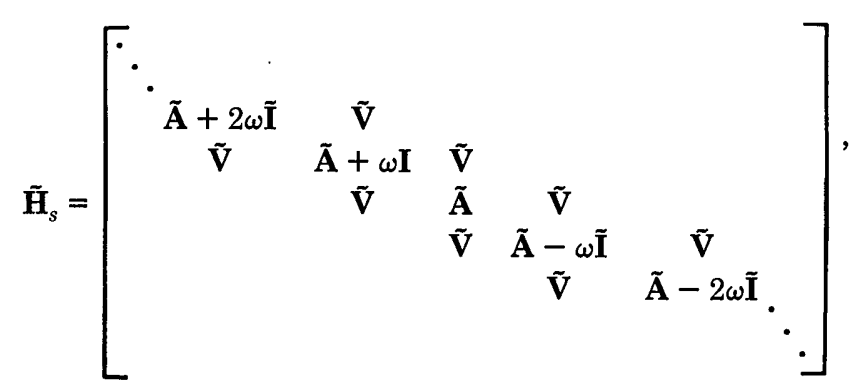

where $\tilde{\mathbf{A}}$ is the free-atom Hamiltonian matrix in terms of the basis set of Eq. (8), within the same photon number $n$, and $\tilde{\mathbf{V}}$ is the interaction Hamiltonian matrix.

The useful properties of the bound matrix elements are

$$
\begin{aligned}
\left\langle\Psi_{i}(\rho) ; n\left|H_{A}(\rho)+h \omega \alpha_{\omega}{ }^{+} \alpha_{\omega}\right| \Psi_{j}(\rho) ; n^{\prime}\right\rangle= & \left\langle\Psi_{i}(r), n\right| H_{A}(r) \\
& +h \omega \alpha_{\omega}{ }^{+} \alpha_{\omega}\left|\Psi_{j}(r), n^{\prime}\right\rangle \\
= & \left(n h \omega+E_{i} \delta_{i j}\right) \delta_{n n^{\prime}},
\end{aligned}
$$

$$
\begin{aligned}
\left\langle\Psi_{i}(\rho) ; n\right|-\frac{1}{2} z e^{i \theta}\left(\alpha_{\omega}^{+}+\alpha_{\omega}\right) & \left|\Psi_{j}(\rho) ; n \pm 1\right\rangle \\
= & \left\langle\Psi_{i}(r)\left|-\frac{F_{a c}}{2} z\right| \Psi_{j}(r)\right\rangle .
\end{aligned}
$$

Equation (10) implies that, for $\Psi_{i}=\Psi_{0}$, the contribution of its energy $E_{0}$ to the complex energy $z_{0}$ is indeed taken into account in the complex plane.

Each $L^{2}$ scattering state, denoted by $X_{j}(\rho)$, is a vectorcoupled product of a bound correlated core and a Gamow orbital (as yet unspecified) corresponding to each decay channel:

$$
X_{j}(\rho)=X_{j}^{c}(\rho) \otimes \epsilon l(\theta)
$$

The rotated, square-integrable Gamow orbital $\epsilon l(\theta)$ is expanded in terms of a square-integrable basis set with real coordinates $\varphi_{k}(r)$ :

$$
\epsilon l(\theta)=\sum_{k} c_{k} \varphi_{k}(r)
$$

Then the various matrix elements of the Hamiltonian where $X_{i}$ appear are given by

$$
\left\langle X_{i}(\rho) ; n|H(\rho)| X_{j}(\rho) ; n\right\rangle=\left\langle x_{i}^{c}(\rho) \otimes \sum_{k} c_{k} \varphi_{k}(r) ; n|H(\rho)| X_{j}^{c}(\rho)\right.
$$

$$
\begin{aligned}
& \left.\otimes \sum_{m} c_{n} \varphi_{m}(r) ; n\right\rangle \\
= & n h \omega \delta_{i j}+\left\langle X_{i}^{c}(r) \otimes \sum_{k} c_{k} \varphi_{k}\left(r e^{-i \theta}\right)\right. \\
& \left.\times\left|H_{A}(r)\right| X_{j}^{c}(r) \otimes \sum_{m} c_{m} \varphi_{m}\left(r e^{-i \theta}\right)\right\rangle,
\end{aligned}
$$




$$
\begin{aligned}
\left\langle X_{i}(\rho) ; n|H(\rho)| X_{j}(\rho) ; n \pm 1\right\rangle= & \left\langle X_{i}^{c}(\rho) \otimes \sum_{k} c_{k} \varphi_{k}(r) ; n|H(\rho)|\right. \\
& \left.\times X_{j}^{c}(\rho) \otimes \sum_{m} c_{m} \varphi_{m}(r) ; n \pm 1\right\rangle \\
= & \left\langle X_{i}^{c}(r) \otimes \sum_{k} c_{k} \varphi_{k}\left(r e^{-i \theta}\right)\left|-\frac{F_{a c}}{2} z\right|\right. \\
& \left.\times X_{j}^{c}(r) \otimes \sum_{m} c_{m} \varphi_{m}\left(r e^{-i \theta}\right)\right\rangle
\end{aligned}
$$

$\left\langle\Psi_{i}(\rho) ; n|H(\rho)| X_{j}(\rho) ; n \pm 1\right\rangle$

$$
=\left\langle\Psi_{i}(r)\left|-\frac{F_{a c}}{2} z\right| X_{j}^{c}(r) \otimes \sum_{k} c_{k} \varphi_{k}\left(r e^{-i \theta}\right)\right\rangle .
$$

Equations (10)-(13b), which result from the separation [Eq. (8)], show that two types of computation are needed. The first is the calculation of state-specific, correlated functions for the bound states, ${ }^{40}$ and the second is the optimization of the Gamow orbitals in terms of the coefficients $c_{k}$ for each $X_{j}^{c}(r)$ and photon occupation number $n$. The elements of the matrices $\tilde{\mathbf{A}}$ and $\tilde{\mathbf{V}}$ [see Eqs. (9) and (9a)] involve functions of real coordinates-obtained either analytically or numerically-that represent numerical multiconfigurational Hartree-Fock zeroth-order and analytic correlation vectors for the bound-state wave functions $\left[\Psi_{j}(r)\right]$ (Ref. 40) as well as for the core wave functions $\left[X_{j}^{c}(r)\right]$ and rotated basis sets $\varphi_{k}(r)$ in the inverse direction, $\varphi_{k}\left(r e^{-i \theta}\right)$, which must remain square integrable.

The $\theta$ dependence in $\varphi_{k}$ implies the construction of a $\theta$ dependent MEMP matrix. On the other hand, the exact eigenvalue $z_{0}$ [Eqs. (4) and (7)] is $\theta$ independent. A good calculation should thus yield a reasonably large range of complex eigenvalues, corresponding to the initial boundstate $\Psi_{0}$ wave function, which are essentially $\theta$ independent.

In summary, the choice of the $N$-electron basis set of Eq. (8) contains the following advantages, which make the solution of the MEMP problem tractable: The bulk of the many-electron calculations are carried out only once, on the real coordinate axis. ${ }^{17,18,48}$ For the determination of the energy shift and width, the computations in the complex plane involve only small matrices with matrix elements for which only the continuum orbitals are rotated by $\rho=r e^{-i \theta}$. In this way, the dimensions of the $\tilde{\mathbf{A}}$ and $\tilde{\mathbf{V}}$ matrices are reduced to the point at which rigorous calculations on polyelectronic atoms are possible without the use of supercomputers. Finally, the physically motivated choice of the function spaces of Eq. (8) leads naturally to the easy identification of the solution vector, i.e., the one whose overlap with the unperturbed initial-state wave function is adiabatically the largest.

Solution of Eq. (9) by direct diagonalization is inconvenient, especially when the field strength is large. Instead, we have developed a self-consistent iterative method that uses the appropriate recurrence relations and solves Eq. (9) efficiently (see Refs. 17 and 43 and Appendix A). In the case of a $2 \times 2$ matrix, this approach reduces to the theory of Autler and Townes. ${ }^{52}$

\section{RESULTS}

The MEMP theory was applied to two atoms, $\mathrm{H}$ and $\mathrm{Li}^{-}$. Hydrogen was chosen as a test case since it is computable accurately by more-conventional methods. Our results are presented in Table 3, together with those obtained by application of perturbation theory. ${ }^{8,53}$ The agreement is excellent except for the $\gamma(\omega)$ at $\omega=0.0430$ a.u., where a small discrepancy is observed. For the Gamow orbital, angular momenta up to $l=4$ were included [Eq. (11)].

Tables 1, 2, and 4 contain our results for the $\mathrm{Li}^{-} \alpha, \alpha(\omega), \gamma$, and $\gamma(\omega)$. The $\mathrm{Li}^{-}$ground-state wave function contains 43 terms, of which the zeroth order was obtained numerically at the multiconfigurational Hartree-Fock level:

$$
\Psi_{0}^{\mathrm{MCHF}}=0.9328\left(1 s^{2} 2 s^{2}\right)+0.3605\left(1 s^{2} 2 p^{2}\right),
$$

whereas the remaining correlation vectors were obtained by minimizing the total energy. ${ }^{40}$ The resulting energy is $E_{0}=$ -7.455364 a.u. and compares well with the configuration interaction result of Weiss, ${ }^{54} E=-7.4553$ a.u. (Our $\mathrm{Li}^{-}$ function is better than the one used before. ${ }^{18}$ )

Two Li thresholds were included in the MEMP matrix, the $1 s^{2} 2 s$ and the $1 s^{2} 2 p$ configurations, with $E_{1}=-7.432726$ a.u. and $E_{2}=-7.36507$ a.u., respectively. The coupling of the ground state $\psi_{0}$ through the dipole field was as follows:

$$
\begin{aligned}
& \Psi_{0} \stackrel{F z}{\rightarrow} 1 s^{2} 2 s \epsilon l, \quad l=0,1, \ldots, 10, \quad \epsilon l=r^{n} \exp \left(-\alpha r e^{-i \theta}\right), \\
& \pi / 2<\theta<0, \\
& \stackrel{F z}{\rightarrow} 1 s^{2} 2 p \epsilon s{ }^{1} P^{\circ}, 1 s^{2} 2 p \epsilon p{ }^{1} S,{ }^{1} P,{ }^{1} D, 1 s^{2} 2 p \epsilon d^{1} P^{\circ},{ }^{1} D^{\circ},{ }^{1} F^{\circ} \text {. }
\end{aligned}
$$

We have already mentioned the importance of including many angular momenta for the free electron in the final state, ${ }^{17,18}$ a necessity that our MEMP theory can handle. For example, in the present $\mathrm{Li}^{-}$case, when the expansion is truncated to ${ }^{1} S$ and ${ }^{1} P^{\circ}$ symmetries only, with $s$ and $p$ orbitals (i.e., $\psi_{0}, 1 s^{2} 2 s \epsilon s, \epsilon p, 1 s^{2} 2 p \epsilon s, \epsilon p$ ), the static polarizabilities become $\alpha=585.3$ a.u. and $\gamma=2.8 \times 10^{8}$ a.u. Note that this value of $\alpha$ is close to the coupled-cluster singles and doubled polarization propagator approximation (CCSDPPA) result of Canuto et al. ${ }^{24}$

Table 3. Linear and Nonlinear Polarizabilities of the Hydrogen Atom (a.u.)

\begin{tabular}{lll}
\hline$\omega$ (a.u.) & $\alpha(\omega)$ & \multicolumn{1}{c}{$\gamma(\omega)$} \\
\hline \multirow{2}{*}{0.00} & $4.500^{a}$ & $1331.3^{a}$ \\
& $4.500^{b}$ & $1333.125^{b}$ \\
\multirow{2}{*}{0.0430} & $4.550^{a}$ & $2151.9^{a}$ \\
& $4.550^{c}$ & $2264.2^{c}$ \\
0.0656 & $4.618^{a}$ & $2380.3^{a}$ \\
& $4.618^{c}$ & $2381.6^{c}$ \\
\hline
\end{tabular}

\footnotetext{
${ }^{a}$ MEMP theory, this work.

${ }^{b}$ Group theoretic implementation of perturbation theory. ${ }^{52}$

c Perturbation theory in the quasi-energy formalism. ${ }^{8}$
} 
Table 4. Linear and Nonlinear Polarizabilities of $\mathrm{Li}^{-}$(a.u.) from the Present MEMP Theory ${ }^{a}$

\begin{tabular}{|c|c|c|c|c|c|c|c|}
\hline$\omega$ (a.u.) & $\alpha(\omega)$ & $\omega(\mathrm{a} . u)$ & $\alpha(\omega)$ & $\omega$ (a.u.) & $\gamma(\omega)$ & $\omega(\mathrm{a} . \mathrm{u})$ & $\gamma(\omega)$ \\
\hline 0.000 & 748.5 & 0.052 & -196.3 & 0.000 & $5.1(8)$ & 0.056 & $-1.8(7)$ \\
\hline 0.002 & 750.6 & 0.054 & -219.4 & 0.002 & $5.3(8)$ & 0.058 & $-1.7(7)$ \\
\hline 0.004 & 756.8 & 0.056 & -236.7 & 0.004 & $5.8(8)$ & 0.060 & $-1.1(7)$ \\
\hline 0.006 & 767.6 & 0.058 & -249.5 & 0.006 & $6.7(8)$ & 0.062 & $-1.2(7)$ \\
\hline 0.008 & 783.3 & 0.060 & -258.7 & 0.008 & $8.7(8)$ & 0.064 & $-9.3(6)$ \\
\hline 0.010 & 805.0 & 0.062 & -265.0 & 0.010 & $1.5(9)$ & 0.066 & $-7.3(6)$ \\
\hline 0.012 & 833.9 & 0.064 & -269.2 & 0.012 & $1.7(9)$ & 0.068 & $-4.8(6)$ \\
\hline 0.014 & 872.1 & 0.066 & -271.7 & 0.014 & $-1.5(8)$ & 0.082 & $5.1(6)$ \\
\hline 0.016 & 923.3 & 0.068 & -272.9 & 0.016 & $-8.7(8)$ & 0.086 & $4.2(6)$ \\
\hline 0.018 & 994.1 & 0.070 & -273.1 & 0.018 & $-1.4(9)$ & 0.088 & $8.0(6)$ \\
\hline 0.020 & 1098.5 & 0.072 & -272.8 & 0.020 & $-1.9(9)$ & 0.090 & $-2.3(7)$ \\
\hline 0.022 & 1281.8 & 0.074 & -272.2 & 0.022 & $-3.1(9)$ & 0.092 & $-2.7(7)$ \\
\hline 0.024 & 1658.4 & 0.076 & -271.6 & 0.024 & $-2.7(9)$ & 0.094 & $6.1(6)$ \\
\hline 0.026 & 1654.7 & 0.078 & -271.3 & 0.028 & $1.0(9)$ & 0.096 & $1.1(7)$ \\
\hline 0.028 & 1438.7 & 0.080 & -271.7 & 0.030 & $1.1(9)$ & 0.098 & $1.2(7)$ \\
\hline 0.030 & 1160.9 & 0.082 & -273.5 & 0.032 & $8.7(8)$ & 0.100 & $8.7(6)$ \\
\hline 0.032 & 891.9 & 0.084 & -277.4 & 0.034 & $5.7(8)$ & 0.102 & $8.7(6)$ \\
\hline 0.034 & 657.1 & 0.086 & -285.3 & 0.036 & $3.6(8)$ & & \\
\hline 0.036 & 460.4 & 0.088 & -301.4 & 0.038 & $2.2(8)$ & & \\
\hline 0.038 & 299.9 & 0.090 & -351.1 & 0.040 & $1.3(8)$ & & \\
\hline 0.040 & 171.0 & 0.092 & -426.6 & 0.042 & $6.7(7)$ & & \\
\hline 0.042 & 68.3 & 0.094 & -407.9 & 0.044 & $2.7(7)$ & & \\
\hline 0.044 & -13.1 & 0.096 & -383.2 & 0.048 & $-1.2(7)$ & & \\
\hline 0.046 & -77.0 & 0.098 & -358.6 & 0.050 & $-1.6(7)$ & & \\
\hline 0.048 & -127.1 & 0.100 & -335.5 & 0.052 & $-1.9(7)$ & & \\
\hline 0.050 & -166.1 & 0.102 & -314.2 & 0.054 & $-1.7(7)$ & & \\
\hline
\end{tabular}

a The numbers in parentheses indicate powers of 10.

It is obvious from Table 4 that $\alpha(\omega)$ goes through a maximum and a minimum (the one-photon ionization starts at $\omega$ $=0.0224$ a.u.). Its shape is similar to the one computed by Delone et $a l .55$ from a quasi-classical model with a simple wave function, but the absolute numbers differ (for example, the maxima differ by a factor of 2). Such qualitative agreement between the MEMP theory and previous models of negative ions exposed in an ac field was also obtained for the ionization cross sections. ${ }^{18}$

Finally, with respect to $\gamma(\omega)$, this is the first prediction to our knowledge for a polyelectronic $(N>2)$ negative ion. It is interesting to observe that now there are two maxima.

\section{SYNOPSIS}

The possibility of theoretical prediction of the dynamic nonlinear polarization of atoms and molecules is a serious challenge to the quantum theory of many-electron systems in ground or excited states. The significance of this statement can be deduced from the discussion in Sections 1 and 2, where a large number of references were made to the advanced methods of treating this problem, with emphasis on the highly polarizable negative ions.

We have presented and applied a MEMP theory for the reliable computation of nonlinear static and dynamic polarizabilities. The theory goes beyond the lowest order of perturbation expansion in the external field as well as in the interelectronic interaction. Thus the polarizabilities of interest are obtained as coefficients of the time-averaged Taylor series of the induced total energy shift $\Delta(\omega) . \quad \Delta(\omega)$ is deduced from a complex eigenvalue that is the solution of an appropriately constructed and efficiently solved non-Hermitian MEMP matrix.

The first application of the MEMP theory was made to a negative ion, $\mathrm{Li}^{-}$. This system offers the opportunity for a good test of any advanced theory, since its number of electrons is small and yet its structure and spectrum are not simple. For the static, linear polarizability $\alpha$, our result agrees well with a recent full $\mathrm{Cl}$ calculation (see Ref. 24 and Table 1). Also, our $\alpha(\omega)$ values verify the qualitative behavior predicted earlier from a semiclassical theory. ${ }^{18,55}$ With respect to the nonlinear polarizabilities, $\gamma$ and $\gamma(\omega)$, which are much more cumbersome to compute, the present results constitute the first predictions of which we are aware.

\section{APPENDIX A}

In the text we state that the solution of the eigenvalue Eq. (9) by direct diagonalization is intractable, especially when the field strength is large, since it is necessary to diagonalize a large portion of the infinite MEMP matrix $\tilde{H}_{s}(\theta)$ in order to include all significant ${ }^{56}$ large-order contributions to the energy shift [see Eqs. (1) and (3)]. This bottleneck is avoided by transforming the eigenvalue Eq. (9) into an infinite set of equations:

$$
\tilde{\mathbf{V}} \mathbf{X}_{n-1}(\theta)+(\tilde{\mathbf{A}}+n \omega \tilde{\mathbf{I}}) \mathbf{X}_{n}(\theta)+\tilde{\mathbf{V}} \mathbf{X}_{n+1}(\theta)=z_{0} \mathbf{X}_{n}(\theta)
$$

where $n$ runs from $-\infty$ to $+\infty, \tilde{\mathbf{I}}$ is the unit matrix, $z_{0}$ is the desired eigenvalue, and $\mathbf{X}_{n}(\theta)$ is the part of the complexified eigenvector corresponding to photon number $n$.

The next step is the introduction of the shift matrices $\tilde{\mathbf{P}}_{n}$ and $\tilde{\mathbf{Q}}_{n}$ with the properties 


$$
\mathbf{X}_{n}=\tilde{\mathbf{P}}_{n} \mathbf{X}_{n+1}, \quad \mathbf{X}_{n}=\tilde{\mathbf{Q}}_{n} \mathbf{X}_{n-1},
$$

which are used in Eq. (A1) to obtain the following recurrence relations for $\tilde{\mathbf{P}}_{n}$ and $\tilde{\mathbf{Q}}_{n}$ :

$$
\begin{gathered}
\tilde{\mathbf{P}}_{n}=-\frac{\tilde{\mathbf{I}}}{\tilde{\mathbf{A}}+n \omega \tilde{\mathbf{I}}-z_{0} \tilde{\mathbf{I}}+\tilde{\mathbf{V}} \tilde{\mathbf{P}} \tilde{n}_{n-1}} \tilde{\mathbf{V}}, \\
\tilde{\mathbf{Q}}_{n}=-\frac{\tilde{\mathbf{I}}}{\tilde{\mathbf{A}}+\mathbf{n} \omega \tilde{\mathbf{I}}-z_{0} \tilde{\mathbf{I}}+\tilde{\mathbf{V}} \tilde{\mathbf{Q}}_{n+1}} \tilde{\mathbf{V}} .
\end{gathered}
$$

At this point, the eigenvalue problem of Eq. (9) is reduced to the solution of the finite-dimension eigenvalue equation [Eq. (A1), $n=0$ ]:

$$
\tilde{\mathbf{V}} \mathbf{X}_{-1}+\tilde{\mathbf{A}} \mathbf{X}_{0}+\tilde{\mathbf{V}} \mathbf{X}_{1}=z_{0} \mathbf{X}_{0}
$$

or

$$
\left(\tilde{\mathbf{A}}+\tilde{\mathbf{V}} \tilde{\mathbf{P}}_{-1}+\tilde{\mathbf{V}} \tilde{\mathbf{Q}}_{1}\right) \mathbf{X}_{0}=z_{0} \mathbf{X}_{0} .
$$

The matrices $\tilde{\mathbf{P}}_{-1}$ and $\tilde{\mathbf{Q}}_{1}$ are infinite continued fractions, as can be seen from Eq. (A3), with $n$ extended to $-\infty$ and $+\infty$, respectively.

Now the problem is focused on the self-consistent solution of Eq. (A5) since the matrices $\tilde{\mathbf{P}}_{-1}$ and $\tilde{\mathbf{Q}}_{1}$ depend on the eigenvalue $z_{0}$. The infinite continued fractions of $\tilde{\mathbf{P}}_{-1}$ and $\tilde{\mathbf{Q}}_{1}$ are truncated at some large value of $N$, for which practically

$$
\tilde{\mathbf{P}}_{|N|-1}=\tilde{\mathbf{Q}}_{|N|+1}=0
$$

and after which the final results remain stable.

The above method for solving the MEMP complex eigenvalue matrix thus has the significant advantage that, at the end, the required diagonalizations involve only the small $\tilde{\mathbf{A}}$ and $\tilde{\mathbf{V}}$ matrices, into which all the the essential information is incorporated.

Note added in proof: In a recent publication, ${ }^{57}$ Agren et $a l$. presented results on $\alpha$ and $\alpha(\omega)$ for $\mathrm{Li}^{-}$from multiconfiguration linear response theory and exact full configuration interaction calculations. Their results go up to $\omega=0.023$ a.u. and are in agreement with ours.

\section{REFERENCES AND NOTES}

1. H. J. Silverstone, Phys. Rev. A 18, 1853 (1978); Int. J. Quantum Chem. 29, 261 (1986); J. N. Silverman and D. Bishop, Phys. Rev. A 34, 5142 (1986); J. N. Silverman and J. Hinze, Phys. Rev. A 37, 1208 (1988); L. Pan, K. T. Taylor, and C. W. Clark, Phys. Rev. Lett. 61, 2673 (1988).

2. V. Franceschini, V. Grecchi, and H. J. Silverstone, Phys. Rev. A 32, 1338 (1985); J. N. Silverman and C. A. Nicolaides, Chem. Phys. Lett. 153, 61 (1988).

3. A. D. Buckingham, Adv. Chem. Phys. 12, 107 (1967).

4. P. W. Langhoff, S. T. Epstein, and M. Karplus, Rev. Mod. Phys. 44, 602 (1972).

5. M. N. Grasso, K. T. Chung, and R. P. Hurst, Phys. Rev. 167, 1 (1968).

6. B. J. Orr and J. F. Ward, Mol. Phys. 20, 513 (1971).

7. J. S. Sims and J. R. Rumble, Jr., Phys. Rev. A 8, 2231 (1973).

8. N. L. Manakov, V. D. Ovsynnikov, and L. P. Rapoport, Sov. Phys. JETP 43, 885 (1976).

9. R. M. Glover and F. Weinhold, J. Chem. Phys. 65, 4913 (1976).

10. D. R. Beck and C. A. Nicolaides, Chem. Phys. Lett. 48, 135 (1977).

11. A. J. Sadlej, Int. J. Quantum Chem. 23, 147 (1983).

12. T. F. Jiang and A. F. Starace, Phys. Rev. A 38, 2347 (1988).
13. H. D. Cohen, J. Chem. Phys. 43, 3558 (1965).

14. For large systems it is possible to improve the performance of the independent-particle model, provided that emphasis is given to property-specific basis sets. See the study of molecular $\alpha$ and $\gamma$ in Ref. 15 and subsequent papers that use the same method.

15. C. A. Nicolaides, M. Papadopoulos, and J. Waite, Theor. Chim. Acta 61, 427 (1982); M. Papadopoulos, J. Waite, and C. A. Nicolaides, J. Chem. Phys. 77, 2527 (1982); J. Waite, M. Papadopoulos, and C. A. Nicolaides, J. Chem. Phys. 77, 2536 (1982).

16. D. R. Beck and C. A. Nicolaides, Chem. Phys. Lett. 49, 357 (1977).

17. Th. Mercouris and C. A. Nicolaides, J. Phys. B 21, L285 (1988); "Laser ionization of $\mathrm{H}^{-}$in the presence of a dc field," submitted to J. Phys. B.

18. C. A. Nicolaides and Th. Mercouris, Chem. Phys. Lett. 159, 45 (1989).

19. R. P. M. McEachran, A. D. Stanffer, and S. Greita, J. Phys. B 12, 3119 (1979).

20. H. P. Roy and A. K. Bhattacharya, Mol. Phys. 31, 649 (1976).

21. G. H. F. Diercksen and A. J. Sadlej, Chem. Phys. 131, 215 (1989).

22. M. Jaszunski and B. O. Roos, Mol. Phys. 52, 1209 (1984).

23. J. G. Jelatis, J. Appl. Phys. 19, 419 (1948).

24. S. Canuto, W. Duch, J. Geertsen, F. Müller-Plathe, J. Oddershede, and G. E. Scuseria, Chem. Phys. Lett. 147, 435 (1988).

25. I. R. Epstein, J. Chem. Phys. 53, 1881 (1970).

26. R. L. Graham and D. L. Yeager, Int. J. Quantum Chem. 31, 99 (1987).

27. G. H. F. Diercksen and A. J. Sadlej, Chem. Phys. 77, 429 (1983).

28. H.-J. Werner and W. Meyer, Phys. Rev. A 13, 13 (1976); E.-A. Reinsch and W. Meyer, Phys. Rev. A 18, 1793 (1978).

29. T. Voegel, J. Hinze, and F. Tobin, J. Chem. Phys. 70, 1107 (1979).

30. S. A. Kucharski, Y. S. Lee, G. D. Purvis III, and R. J. Bartlett, Phys. Rev. A 29, 1619 (1984).

31. I. Cernusak, G. H. F. Dierksen, and A. J. Sadlej, Phys. Rev. A 33, 814 (1986).

32. G. Maroulis and A. J. Thakkar, Chem. Phys. Lett. 156, 87 (1989); A. J. Thakkar, Phys. Rev. A 40, 1130 (1989).

33. A. Kumar and W. J. Meath, Can. J. Chem. 63, 1616 (1985).

34. D. M. Bishop and B. Lam, Phys. Rev. A 37, 464 (1988).

35. L. L. Boyle, A. D. Buckingham, R. L. Disch, and D. A. Dunmur, J. Chem. Phys. 45, 1318 (1966).

36. A. D. Buckingham and P. G. Hibbard, Symp. Faraday Soc. 2, 41 (1968).

37. D. P. Shelton, Phys. Rev. Lett. 62, 2660 (1989).

38. V. Kellö, B. O. Roos, and A. J. Sadlej, Theor. Chim. Acta 74, 185 (1988).

39. P. W. Fowler and P. A. Madden, Phys. Rev. B 29, 1035, 6131 (1984).

40. C. A. Nicolaides, in Quantum Chemistry-Basic Aspects, Actual Trends, R. Carbo, ed. (Elsevier, New York, 1989), p. 343.

41. In Ref. 10 the Fermi-Sea multireference zeroth-order space (which for atoms or diatomics is obtained numerically) was introduced so that dipole polarization-related as well as excitedstate correlations could be built on it. Multireference spaces were also implemented more recently by a number of research. ers in advanced computational methods based on single orthonormal basis sets.

42. For literature relating $\gamma(\omega)$ to the independent components of the nonlinear susceptibility tensor that enter into the determination of measurements (Kerr effect, third-harmonic generation, etc.) see Refs. 6, 8, 28, and 30.

43. C. A. Nicolaides and Th. Mercouris, in Atoms in Strong Fields, C. A. Nicolaides, C. Clark, and M. Nayfeh, eds. (Plenum, New York, to be published).

44. C. A. Nicolaides, Y. Komninos, and Th. Mercouris, Int. J. Quantum Chem. S15, 355 (1981).

45. C. Cerjan, R. Hedges, C. Holt, W. P. Reinhardt, K. Scheibner, and J. J. Wendoloski, Int. J. Quantum Chem. 14, 393 (1978).

46. S.-I. Chu and W. P. Reinhardt, Phys. Rev. Lett. 39, 1195 (1977).

47. A. M. Dykhne and A. V. Chaplik, Sov. Phys. JETP 13, 1002 (1961)

48. C. A. Nicolaides and D. R. Beck, Phys. Lett. 65A, 11 (1978); Int. J. Quantum. Chem. 14, 457 (1978).

49. C. A. Nicolaides and Th. Mercouris, Phys. Rev. A 36, 390 (1987). 
50. The Hamiltonian matrix of Eq. (9a) was deduced by Shirley ${ }^{51}$ from semiclassical theory using the Floquet theorem.

51. J. H. Shirley, Phys. Rev. 138, B979 (1965).

52. S. H. Autler and C. H. Townes, Phys. Rev. 100, 703 (1955).

53. S. P. Alliluev and I. A. Malkin, Sov. Phys. JETP 39, 627 (1974).

54. A. W. Weiss, Phys. Rev. 166, 70 (1968).

55. N. B. Delone, P. A. Golovinsky, I. Y. Keyan, V. P. Krainov, and A. I. Tuguchev, J. Phys. B 19, 2457 (1986).
56. The basis set that is used to construct the matrices $\tilde{\mathbf{A}}$ and $\tilde{\mathbf{V}}$ (see the Results section) is such that it ensures the possibility of taking into account large-order contributions to the energy shift.

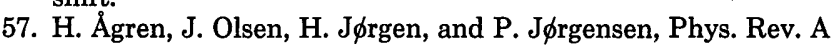
40, 2265 (1989). 